\title{
Multiple rings in a 3D anisotropic Wigner crystal: Structural and dynamical properties
}

\author{
S. W. S. Apolinario, ${ }^{*}$ B. Partoens, ${ }^{\dagger}$ and F. M. Peeters ${ }^{\ddagger}$ \\ Departement Fysica, Universiteit Antwerpen, Groenenborgerlaan 171, B-2020 Antwerpen, Belgium
}

(Received 18 October 2007; published 16 January 2008)

\begin{abstract}
The structural and dynamical properties of small three-dimensional (3D) anisotropically confined Wigner crystals of particles interacting via a Coulombic interparticle potential are investigated. Varying the degree of anisotropy of the confinement potential drives the system from a 3D to a one-dimensional configuration intermediated by a sequence of first and second order structural phase transitions. We classified the ground state configurations with respect to their symmetry resulting in three different groups of configurations: multiple ring, degenerate multiple ring, and nonsymmetric structures. The results are summarized in a phase diagram where the different configurations are identified for systems ranging from $N=4$ to 25 particles. The behavior of the ground state symmetry of large systems was investigated for the cases of $N=50,60$, and 70 particles. A normal mode analysis reveals that multiple ring structures exhibit inter-ring and/or vortex/ antivortex excitation modes of oscillations.
\end{abstract}

DOI: $10.1103 /$ PhysRevB.77.035321

PACS number(s): 64.60.-i, 63.22.-m

\section{INTRODUCTION}

Finite systems of classical charged particles have been of considerable interest, since they can model a variety of systems as electrons trapped on top of liquid helium, ${ }^{1}$ electrons trapped in quantum well structures, ${ }^{2}$ strongly coupled radiofrequency dusty plasmas, ${ }^{3}$ vortex clusters in an isotropic superfluid, ${ }^{4}$ laser-cooled trapped ion systems, ${ }^{5,6}$ complex dusty plasmas, ${ }^{7}$ etc.

Complex plasmas have been typically generated through experiments in parallel plate radio-frequency (rf) discharge. Large particles, called dust particles, introduced into the plasma chamber become electrically charged. A strong upward electric field is used to compensate the downward gravitational force acting on the dust particles. Consequently dust particles are able to levitate and form flat layers of crystalline particles at low temperature and large density. These nearly two-dimensional systems have similarities to colloidal suspensions ${ }^{8,9}$ and show a rich set of interesting phenomena, such as phase transitions,,${ }^{10,11}$ fluid motion, ${ }^{12}$ and a variety of wave phenomena. ${ }^{13}$ By means of suitable horizontal barriers the generation of two-dimensional finite dust clusters is possible. ${ }^{14,15}$

Gravitational effects were circumvented in experiments under microgravity conditions in space ${ }^{16,17}$ or on parabolic flights. ${ }^{18,19}$ Consequently extended three-dimensional complex plasmas could be generated. However, the dust is not evenly distributed in the complete volume, but is disturbed by a dust-free region in the center of the discharge, the socalled void phenomenon. ${ }^{16,18}$

The first experimental investigation on small size spherical three-dimensional homogeneous dust plasma crystals consisting of micrometer-sized polymer particles was carried out recently. ${ }^{7}$ In the latter work, a thermophoretic force was applied to compensate for gravity, which in combination with the plasma induced electric field force establishes a vertical confinement. Such systems were named "Coulomb balls." Theoretical investigation of structural properties and melting behavior in three-dimensional (3D) Coulomb balls are reported in Refs. 20-22, 24, and 25. It was found that inside a Coulomb ball the dust particles are arranged in nested shells. Within each shell the particles form a hexagonal lattice, which is affected by defects as a consequence of the curvature of the shell surface. The large size of the dust particles allows direct observation by simple video microscopy because dynamical processes occur on typical frequency scales of a few hertz, quite unlike strongly coupled colloidal suspensions, ${ }^{23}$ where particle motion is heavily damped. Therefore, this system is ideally suited for studying the static and dynamics of strongly coupled matter with "atomic resolution."

Recent developments of experiments in spherically confined dust plasma have motivated two of our most recent works. $^{24,25}$ Reference 24 presented a detailed numerical simulation of the configuration of the ground state and the lowest-energy metastable state, and of the spectrum of normal modes of classical 3D clusters with isotropic parabolic confinement. The confined particles were considered to interact through a repulsive potential such as Coulomb or screened Coulomb interparticle potential. We found that both small and larger systems satisfy Euler's theorem and the total topological charge defect is 12 . The breathing and the center of mass mode frequencies were analytically determined. The breathing mode was found to have the highest frequency for the case of a Coulomb interparticle interaction potential. Furthermore we found a relation between the appearance of maximum and minimum in the lowest nonzero eigenfrequency and in the second derivative of the binding energy as function of $N$ for small clusters $(N<23)$ from which we determine the most stable and least stable clusters. A thermodynamic investigation of such a system was given in Ref. 25 . It was found that the ground state (GS) configuration of systems with $N=6,12,13$, and 38 particles have large mechanical stability and were therefore identified as magic clusters. The common characteristic of magic clusters is that they are formed by one of the highly regular crystal structures, i.e., an octahedron or icosahedron.

In any real experimental system the confinement potential will have an asymmetric component. Therefore, in this paper we study the dynamical and statical properties of anisotrop- 
ically confined Wigner crystals. Such an investigation is an extension of our previous work ${ }^{24}$ and has relation to previous work on anisotropic confined two-dimensional (2D) clusters. ${ }^{26,27}$ Additionally, our study complements previous investigations of structural phase transitions arising between a $2 \mathrm{D}$ to a $3 \mathrm{D}$ classical artificial atom. ${ }^{29}$ The latter reference showed that the development from a ring structure into a shell configuration passes necessarily though a layered structure which is intermediated by structural transitions of first and second orders. We shall demonstrate that anisotropic finite 3D Wigner crystals form, in fact, a hybrid element in the sense that they exhibit physical properties inherent of both 2D and 3D systems. Toward this objective we determine the lowest-energy configurations and perform a normal mode analysis where we give special attention to delocalized modes, i.e., the normal mode oscillations with very low frequencies.

The present paper is organized as follows. In Sec. II we present our theoretical model. In Sec. III we give our results for the statistical properties of the ground state configurations, which is divided in four subsections: (A) we investigate the structural phase transitions of first and second orders which take place in a small system with $N=6$ particles when the anisotropy parameter is varied, (B) a larger system with $N=18$ particles is investigated and we introduce the fraction of degenerate frequencies $\eta$ as a quantity to characterize highly symmetric clusters, (C) we generalize our results by presenting a phase diagram for systems with $N=4-25$ particles, and (D) larger systems are investigated, i.e., systems with $N=50,60$, and 70 particles. A normal mode analysis of multiple ring structures is discussed in Sec. IV. Finally, in Sec. V we present our conclusions.

\section{THEORETICAL MODEL}

We study a 3D model system of $N$ equally charged particles in an anisotropic confinement potential and interacting through a repulsive potential. The potential energy of the system is given by

$$
E=\sum_{i=1}^{N} \frac{1}{2} m \omega_{0}^{2}\left(x_{i}^{2}+y_{i}^{2}+\alpha z_{i}^{2}\right)+\sum_{i>j}^{N} \frac{q^{2}}{\epsilon_{0}\left|\mathbf{r}_{i}-\mathbf{r}_{j}\right|},
$$

where $\epsilon_{0}$ and $q$ are, respectively, the electric constant and the particle charge, $\mathbf{r}_{i}=\left(x_{i}, y_{i}, z_{i}\right)$ is the coordinate of the $i$ th particle, $N$ is the total number of particles, $\omega_{0}$ is the confinement frequency of a single particle, and $\alpha$ is the anisotropy parameter of the confinement potential. We can write the potential energy (1) in dimensionless form

$$
E=\sum_{i=1}^{N}\left(x_{i}^{2}+y_{i}^{2}+\alpha z_{i}^{2}\right)+\sum_{i>j}^{N} \frac{1}{\left|\mathbf{r}_{i}-\mathbf{r}_{j}\right|},
$$

if we express the coordinate and energy in the following units: $r_{0}=\left(q^{2} / \gamma \epsilon_{0}\right)^{1 / 3}$, where $\gamma=m \omega_{0} / 2$ and $E_{0}=q^{2} / \epsilon_{0} r_{0}$. All our numerical results will be given in dimensionless units.

Intuitively, we expect interesting behavior as a function of $\alpha$ which governs the anisotropy of the confinement potential. This can be seen as follows: for $\alpha=0$ we have an unbounded system in one direction, i.e., a wirelike configuration as discussed in Ref. 28, while for $\alpha=1$ the system consists of an isotropic 3D Wigner crystal. This implies that, as a function of $\alpha$ structural transitions (e.g., configurational changes) have to take place. We found that the ground state configuration shows a surprisingly complex behavior as a function of $\alpha$.

To obtain the stable configurations we use the Monte Carlo simulation technique supplemented with the Newton method in order to speed up the computer program and to increase the accuracy of the found energy value (see Ref. 30 for details). By implementing a large number of different simulations starting from different random initial configurations we are confident that we found the ground state configuration as long as the number of particles $N$ is not too large, i.e., roughly $N<60$. Depending on the total number of particles, between several hundred to several thousand random initial configurations were generated.

The eigenfrequencies are the square root of the eigenvalues of the dynamical matrix

$$
H_{\alpha \beta, i j}=\left.\frac{\partial^{2} H}{\partial r_{\alpha, i} \partial r_{\beta}, j}\right|_{r_{\alpha, i}=r_{\alpha, i}^{n}},
$$

where $\left\{r_{\alpha, i}^{n} ; \alpha=x, y, z ; i=1, \ldots, N\right\}$ are the positions of the particles in a stable configuration.

\section{RESULTS}

$$
\text { A. } N=6
$$

The influence of the anisotropy parameter $\alpha$ of the confinement potential to the GS configuration is investigated in this subsection for a small system. In order to avoid a complicate analysis but still acquire satisfactory knowledge of mechanical processes ${ }^{32}$ induced by the anisotropy parameter we first investigate a relatively small system, i.e., the system with $N=6$ particles. It is already known for some time ${ }^{33}$ that for $\alpha=1$, i.e., for an isotropic confinement potential, the GS configuration of the system with $N=6$ particles forms a magic cluster. Magic clusters present an enhanced mechanical stability which is reflected in a large value of the lowest nonzero eigenfrequency and a pronounced value of the addition energy, which is defined as $\Delta E=E(N+1)+E(N-1)$ $-2 E(N)$ where $E(N)$ is the GS configuration of the system with $N$ particles. ${ }^{24,25,30}$

Figure 1 shows the eigenfrequency spectrum for the system with $N=6$ particles as function of the anisotropy parameter. For $\alpha=1$, i.e., for the isotropic case, we see that the normal modes are highly degenerate. Of the total of 18 normal modes present in the $3 \mathrm{D}$ system with $N=6$ confined particles we found only seven different values for the eigenfrequencies. However, with decreasing value of the anisotropy parameter $\alpha$ the degeneracy is lifted (see Fig. 1). Notice that at $\alpha=0.4445$ the eigenfrequencies undergo a discontinuity. Such discontinuity is found to be a consequence of a structural phase transition, i.e., a configurational change of the GS configuration, induced by the anisotropy parameter. We show the GS configurations for $\alpha=0.444$ and 0.445 , respectively, in the insets I and II of Fig. 2(a). We 


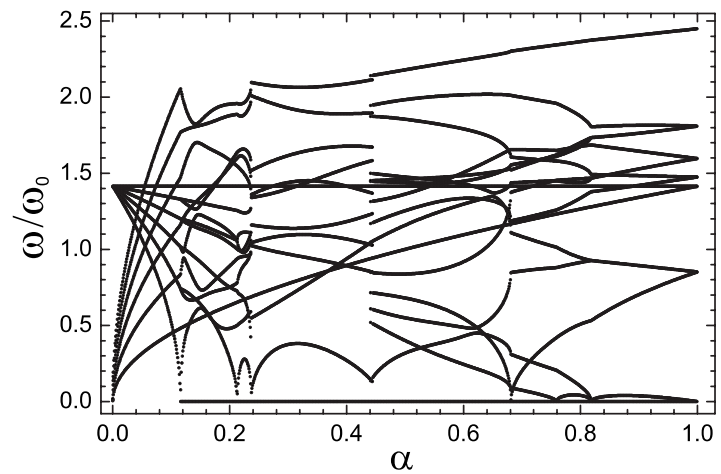

FIG. 1. Normal mode frequencies for the system with $N=6$ particles as function of the anisotropy parameter.

notice that for $\alpha=0.445$ the GS configuration still exhibits a shell structure while for $\alpha=0.444$ this is no longer true. In order to know the nature of the structural transition we calculate the first derivative of the energy with respect to the anisotropy parameter [see Fig. 2(a)]. The derivative of the energy is discontinuous at the critical point $\alpha=0.4445$ which characterizes a structural phase transition of first order. The distribution of particles along the $z$ direction is found to be strongly dependent on the anisotropy parameter near this structural transition. Figure 2(b) displays the usual spherical coordinate $\theta$ of each particle as function of the anisotropy parameter. The number written near each line indicates how many particles have the same value for the spherical coordi-

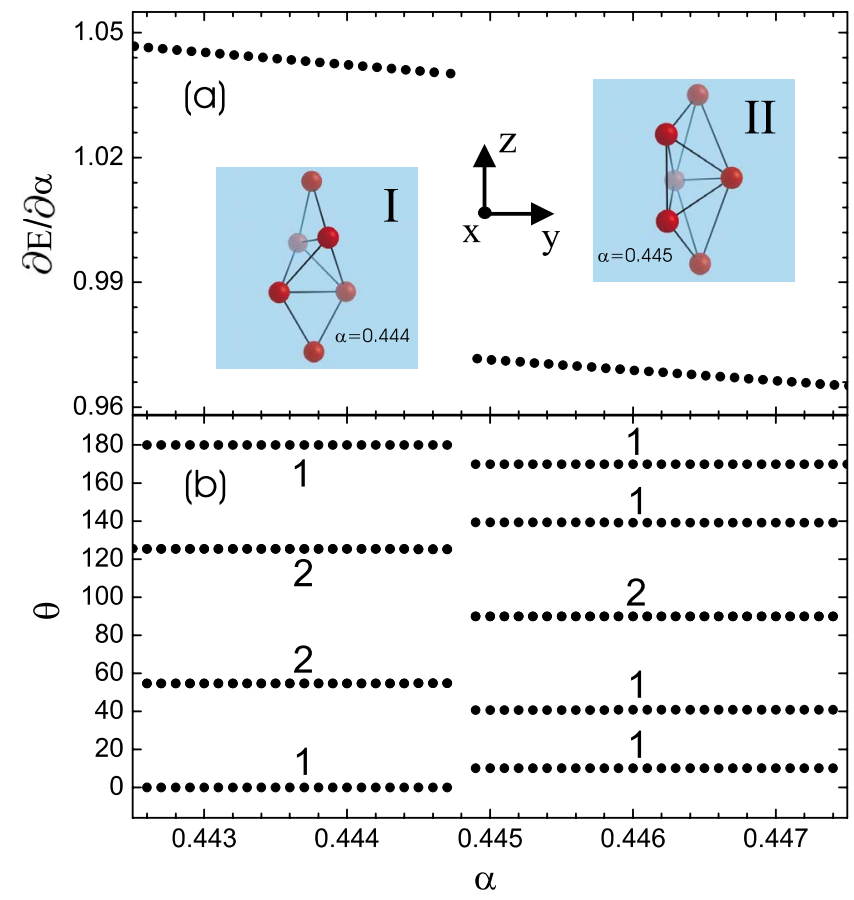

FIG. 2. (Color online) (a) First derivative of the energy $E$ with respect to the anisotropy parameter $\alpha$; insets I and II display, respectively, the GS configuration for $\alpha=0.444$ and 0.445 , i.e., the GS configuration just before and after the critical anisotropy parameter of $\alpha=0.4445$. (b) The spherical coordinate $\theta$ of each particle as function of the anisotropy parameter $\alpha$.

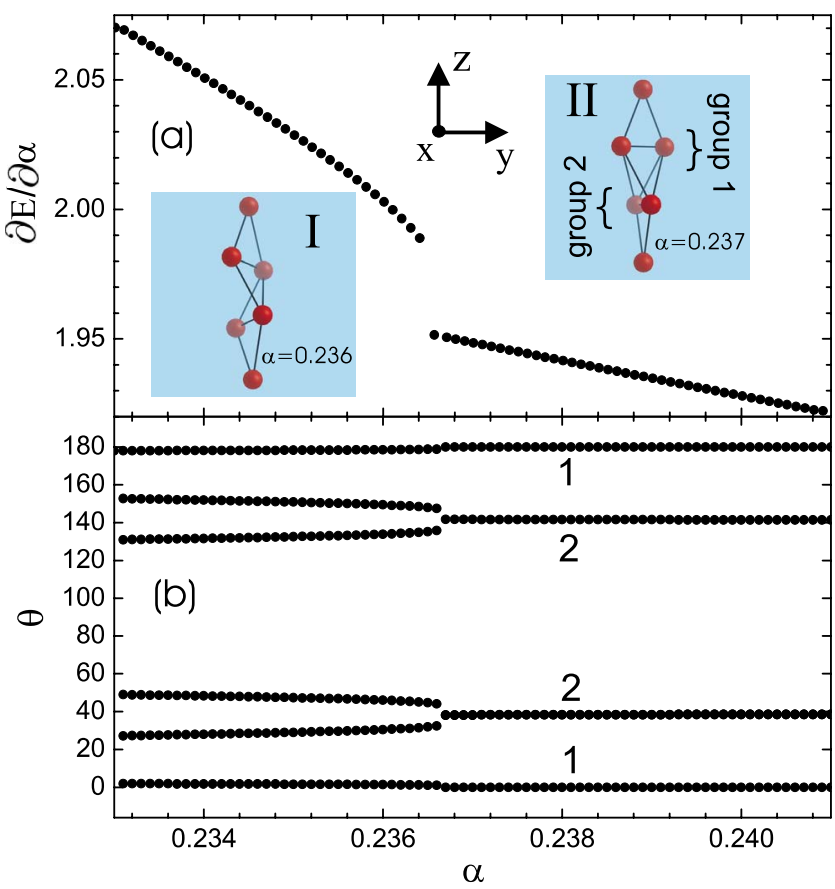

FIG. 3. (Color online) The same as Fig. 2 but for smaller values of the anisotropy parameter, i.e., for $0.233<\alpha<0.241$.

nate. Clearly we see also a pronounced jump in the values of $\theta$ for all particles at the critical anisotropy parameter.

Even for a highly asymmetric confinement potential the system is able to undergo a first order phase transition at $\alpha$ $=0.2365$. However, in this case the GS configuration has almost a one-dimensional structure and the change in the particle's position is much smaller. Figure 3(a) displays the first derivative of the energy with respect to the anisotropy parameter. We can see that the first derivative of the energy is discontinuous at the critical anisotropy parameter of $\alpha$ $=0.2365$. The GS configuration just before the transition, i.e., for $\alpha=0.237$ (see inset II of Fig. 3), is equivalent to the configuration found previously when $\alpha=0.444$ (see inset I of Fig. 2). Particles in such a configuration arrange themselves in the following way: (1) the top and bottom particles are aligned along the $z$ axis, (2) the other four particles form two groups as indicated in the inset II of Fig. 3(a) where particles belonging to the same group have the same value of the $z$ coordinate, and (3) the difference in the usual azimuthal angle of any two particles belonging to distinct groups is always $90^{\circ}$. This type of configuration also appears in larger clusters as we will see later and they have a specific eigenfrequency distribution. This configuration has the arrangement (1:2:2:1) where we account for the particles' distribution along the $z$ direction. For decreasing values of $\alpha$, i.e., for $\alpha<0.237$ [see inset I of Fig. 3(a)], the cluster symmetry is broken and the four particles in the most inner region of the cluster obtain different values for their spherical coordinate $\theta$. Such change in the distribution of particles along the $z$ direction is documented in Fig. 3(b), which displays the spherical coordinate $\theta$ of each particle as function of $\alpha$. The number written near each line indicates the number of particles with the same value of the spherical coordinate $\theta$ for a 

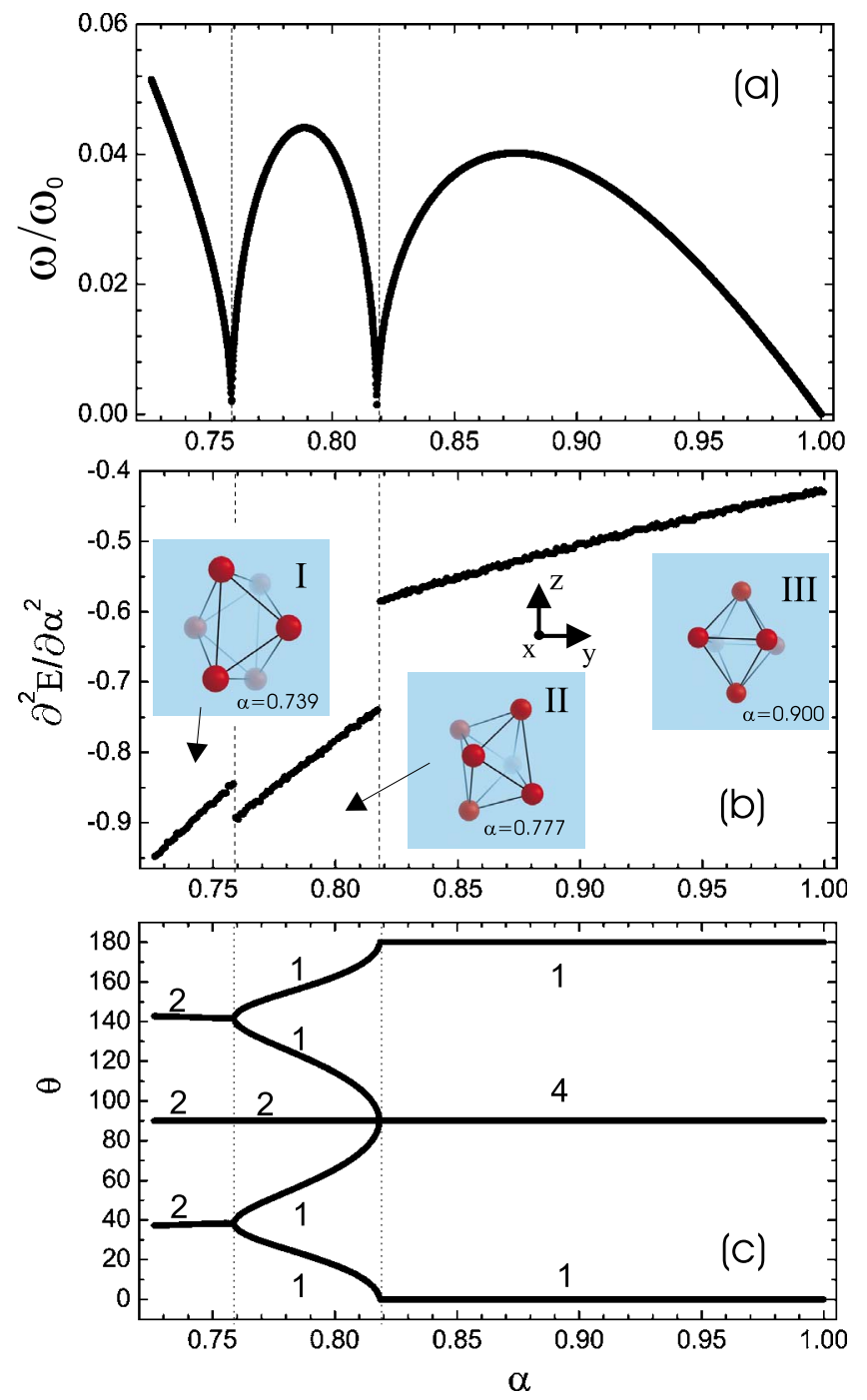

FIG. 4. (Color online) (a) Lowest-energy eigenfrequency for the system with $N=6$ particles as function of the anisotropy parameter. (b) Second derivative of the energy with respect to the anisotropy parameter $\alpha$; insets I, II, and III display, respectively, the GS configurations for $\alpha=0.739,0.777$, and 0.900 . (c) The spherical coordinate $\theta$ of each particle as function of the anisotropy parameter $\alpha$.

given $\alpha$. We can see in Fig. 3(b) that for $\alpha<0.2365$ there are six distinct lines where each one is associated with one particle while for $\alpha>0.2365$, i.e., after the transition point, the number of different lines is reduced and the two most inner curves become associated with two particles.

The anisotropy parameter also drives the system to undergo second order transitions. Such second order phase transitions happen when the critical anisotropy parameter reaches $\alpha=0.818,0.759,0.682,0.212$, and 0.116 . Those second order transitions are found to involve a continuous change in the particle's position and in the eigenfrequencies of the system. Figure 4(a) shows the lowest nonzero frequency as function of the anisotropy parameter in the slightly anisotropic regime, i.e., for $\alpha>0.7$. We can see that at the critical values of the anisotropy parameter of $\alpha=0.818$ and 0.759 the eigenfrequency spectrum exhibits a softening of the lowest-energy mode. For such transition the first deriva- tive of the energy with respect to the anisotropy parameter remains continuous while its second derivative exhibits a discontinuity at the two critical values $\alpha=0.818$ and 0.759 as we see in Fig. 4(b). To help us in understanding the effects of the second order phase transition on the structure of the cluster we display in the insets I, II, and III of Fig. 4(b) the GS configurations found, respectively, for the cases of $\alpha$ $=0.739,0.777$, and 0.9 .

Before any phase transition takes place, i.e., for $\alpha$ $>0.818$, the ground state configuration of the system remains the same and corresponds to an octahedron structure, where its main axis points to the $z$ direction [see, for example, inset III in Fig. 4(b)]. Between the two critical values of the anisotropy parameter, i.e., for $0.759<\alpha<0.818$, we can see that the main axis of the icosahedron deviates from the $z$ direction [see inset II of Fig. 4(b)]. Finally for $\alpha$ $<0.759$ the icosahedron reaches another orientation and its main axis lays on the $x y$ plase. Such change in the orientation of the icosahedron can be better followed from the dependence of the spherical coordinate $\theta$ to the anisotropy parameter [see Fig. 4(c)].

\section{B. $N=18$}

The GS configuration for the system with six particles forms a structure with only one shell. The eigenfrequency spectrum and the structural phase transitions of larger clusters are expected to present a more complex behavior. Now we turn our attention to larger systems and we start our investigation by considering the system with $N=18$ particles having the GS configuration $(1,17)$ when $\alpha=1$ which is different from the simple shell structure, i.e., it has one extra particle in the center. The first nonzero frequency gives us an indication of the structural phase transition undergone by the system as $\alpha$ is changed. Figure 5(a) displays the first nonzero frequency for the system with $N=18$ particles and anisotropy parameter varying from $\alpha=0.15$ to 1 . We notice that there are several discontinuities in the lowest nonzero frequency and they correspond to first order structural transitions, i.e., at $\alpha=0.570,0.491,0.336$, and 0.234 . A second order transition occurs at $\alpha=0.459$, i.e., when a mode softening occurs. Such first and second order structural phase transitions can drive the system to configurations of higher symmetry. The eigenfrequency spectrum must be sensitive to the symmetry of the cluster. Most importantly, the number of degenerate frequencies increases for highly symmetric clusters. We defined for a given value of $\alpha$ and particle number $N$ the fraction of degenerate frequencies $\eta$ which is the ratio between the number of degenerate frequencies and the total number of frequencies, i.e., the $3 N$ frequencies of any given system of $N$ particles. Fig. 5(b) displays $\eta$ as function of the anisotropy parameter $\alpha$ for the system with $N=18$ particles. We notice in Figure 5(b) three regions of $\alpha$ with relatively large $\eta$ value. Those regions are indicated by the blue, yellow, and green areas and correspond, respectively, to the ranges 0.57 $\leqslant \alpha \leqslant 1,0.336 \leqslant \alpha \leqslant 0.434$, and $0.159 \leqslant \alpha \leqslant 0.233$. The values of $\eta$ at those regions are, respectively, equal to $\eta$ $=0.777,0.740$, and 0.518 . Oppositely, the blank areas correspond to the situation of highly nonsymmetric clusters with very low values of $\eta$. 


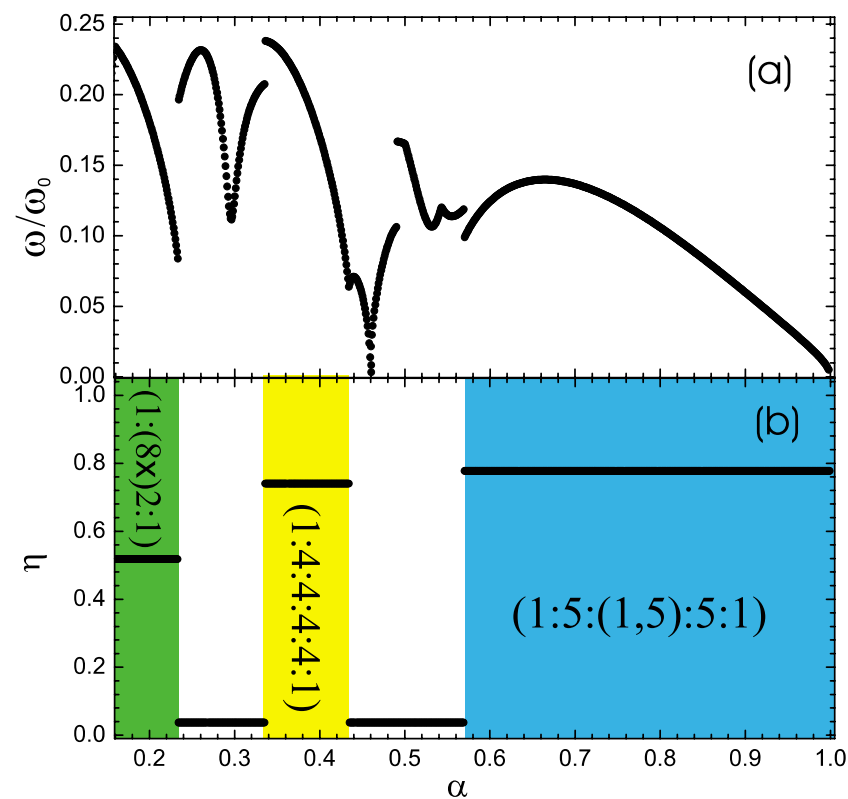

FIG. 5. (Color online) (a) Lowest-energy normal mode frequency for the system with $N=18$ particles as function of the anisotropy parameter $\alpha$. (b) The fraction of degenerate frequencies $\eta$ in the system as function of $\alpha$. The regions of multiple ring structures are indicated by blue and yellow areas and correspond, respectively, to the configurations [1:5: $(1,5): 5: 1]$ and $(1: 4: 4: 4: 4: 1)$. The region corresponding to a degenerate multiple ring structure of the type $[(1:(8 \times) 2: 1]$ is indicated by the green area.

Figure 6(d) shows the GS configuration for the system with $N=18$ particles and anisotropy parameter equal to $\alpha$ $=0.7$. Such configuration is representative for the region of $\alpha$ indicated by the blue area in Fig. 5(b). We can describe this configuration as follows [see Fig. 6(d)]: at the extremes of the cluster there are two isolated particles located along the $z$ axis, one particle in the center and three rings formed by five particles in each ring. Furthermore those rings are parallel to each other and perpendicular to the $z$ axis. We use the following notation for such an arrangement [1:5:(1,5):5:1]. The different ring like layers are separated by ":" while the notation for the ring configurations is as previously used for 2D systems. Such multiple ring structure is also found for other

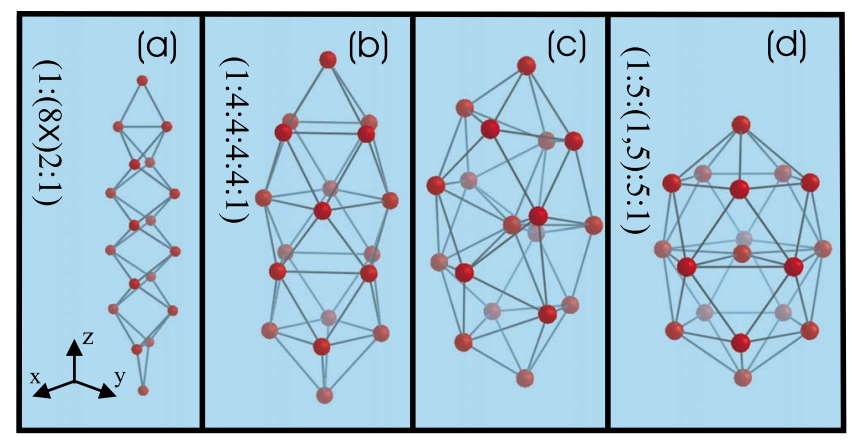

FIG. 6. (Color online) (a), (b), (c), and (d) are the ground state configurations for the system with $N=18$ particles and anisotropy parameter, respectively, equal to $\alpha=0.2,0.4,0.5$, and 0.7 .

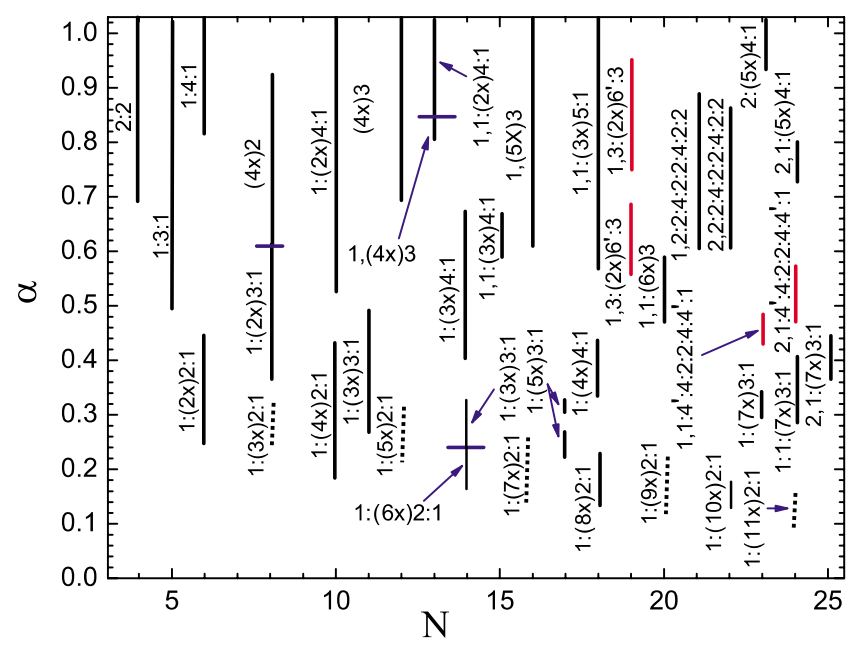

FIG. 7. (Color online) Phase diagram: The vertical lines indicate the values of $\alpha$ where the GS configuration is a multiple ring structure along the $z$ direction. The numbers beside these lines give the particles' GS arrangement.

ranges of the anisotropy parameter. For example, in the yellow region of Fig. 5(b) the multiple ring structure has the arrangement (1:4:4:4:4:1). Differently from the first case, this configuration does not have a particle in the center and is formed by two particles sitting in the extrema of the cluster, and a sequence of four rings with four particles in each ring [see, for example, Fig. 6(b) for $\alpha=0.4$ ]. We observe that those rings have a square shape and are rotated with respect to each other by an angle of $45^{\circ}$. Figure 6(c) shows the GS configuration for $N=18$ particles and $\alpha=0.5$, i.e. a nonsymmetric configuration which is representative for the second blank area in Fig. 5(b). Ultimately, the GS configuration goes to a degenerate multiple ring structure when $0.159 \leqslant \alpha$ $\leqslant 0.233$ (green region). A typical configuration found in this region is shown in Fig. 6(a) for $\alpha=0.2$. This arrangement is similar to the one shown in the inset I of Fig. 2(a) but now there is a sequence of eight sets of two particles. Such arrangement can be referred by the term $(1: 2: 2: 2: 2: 2: 2: 2: 2: 1)$ or shortly $[1:(8 \times) 2: 1]$ where $(8 \times)$ indicates that there are eight sets holding each two particles.

\section{Phase diagram and fine structure}

Such multiple and degenerate multiple rings can be thought as configurations that are intermediate between a two- and three-dimensional configuration. We can say that the multiple ring configuration is a three-dimensional structure formed by smaller two-dimensional parts. Such configuration is not unique to the system with $N=18$ particles but it is also present in other systems. We computed the fraction of degenerate frequencies $\eta$ for all systems ranging from $N=5$ to 25 particles in order to identify the regions of the anisotropy parameter where the system exhibits GS configuration with multiple and/or degenerate multiple ring structures. The result of this investigation is summarized in Fig. 7 in the form of a phase diagram. For each given number of particles $N$ the vertical lines indicate the range of $\alpha$ where the system 


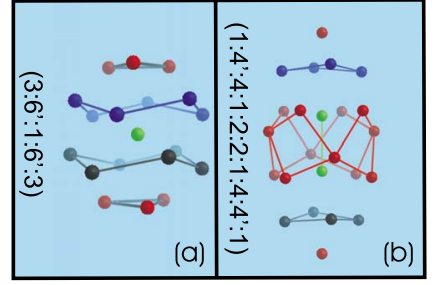

FIG. 8. (Color online) Fine structure for systems with $N=19$ and $\alpha=0.9$ (a) and $N=24$ and $\alpha=0.5$ (b). Particles in blue and black colors form rings with nonzero width. The other particles in the external (inside the external) shell are in red (green) color.

has highly symmetric configurations and beside each vertical line the related particle arrangement is written. The phase diagram shown in Fig. 7 can be divided into two areas, that is, the upper one (roughly $\alpha>0.5$ ) where the multiple rings accommodate about four, five, and six particles; and the lower part (roughly $\alpha<0.5$ ) where we find the degenerate multiple rings and multiple rings with three particles per ring. We noticed that the degenerate multiple ring structures for the systems with an odd number of sets with two particles, i.e., systems with $N=8,12,16,20$, and 24 particles, were not revealed via the computation of $\eta$, although it appeared for systems with an even number of sets of two particles, i.e., systems with $N=6,10,14,18$, and 22 particles. Alternatively, those configurations were obtained by computing the distribution of particles along the $z$ direction and the first nonzero frequency which determined the critical values of $\alpha$ where the system passes through phase transitions. The values of the anisotropy parameter where the degenerate multiple rings occur for the system with $N=8,12,16,20$, and 24 are indicated in Fig. 7 by vertical dotted lines. We notice that only for $N=7$ and 9 particles there are no regions of the anisotropy parameter where high symmetric configurations can be found. The increase of the number of particles leads to the formation of multiple ring configurations where the width of the rings along the $z$ direction is relatively large. Analogous fine structures in 2D systems were reported in Ref. 34. In 3D systems we find that fine structure occurs for systems with $N=19,23$, and 24 particles and anisotropy parameter indicated by vertical red lines in Fig. 7. Examples of such a fine structure is shown in Fig. 8(a) for $N=19$ and $\alpha$ $=0.9$ and Fig. 8(b) for $N=24$ and $\alpha=0.5$. In both cases particles in blue and black colors form rings with nonzero width along the $z$ direction. Other particles, i.e., in the external (inside the external) shell, are represented by red (green) balls. We name the arrangement of Figs. 8(a) and 8(b), respectively, by $\left(3: 6^{\prime}: 1: 6^{\prime}: 3\right)$ and $\left(1: 4^{\prime}: 4: 1: 2: 2: 1: 4: 4^{\prime}: 1\right)$ where the accents refer to rings with nonzero width. The formation of fine structure reduces the interparticle interaction energy of the system. This phenomenon is similar to the zigzag transition induced by the increasing of the particle number. ${ }^{26}$

\section{D. $N=50,60$, and 70}

In order to obtain insight about the particle distribution along the $z$ direction in large clusters we investigated sys-

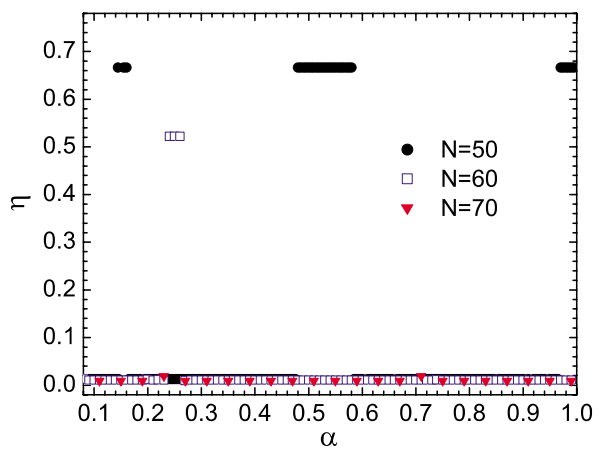

FIG. 9. (Color online) Fraction of degenerate frequencies $\eta$ for the GS configuration of the systems with $N=50,60$, and 70 particles as function of the anisotropy parameter.

tems with $N=50,60$, and 70 particles. In particular, we wish to answer if the GS configuration of large clusters form, symmetric clusters, i.e., multiple or degenerate multiple ring structures. The fraction of degenerate frequencies $\eta$ as function of $\alpha$ is shown in Fig. 9 for the systems with $N=50$ (circle), 60 (square), and 70 (triangle) particles. For the system with $N=50$ particles we can find three distinct regions of the anisotropy parameter, i.e., $0.15 \leqslant \alpha \leqslant 0.16,0.48 \leqslant \alpha$ $\leqslant 0.58$, and $0.98 \leqslant \alpha \leqslant 1.0$, where the value of the fraction of degenerate frequencies is relatively large, i.e., $\eta=0.66$. For other values of the anisotropy parameter the fraction of degenerate frequencies becomes very small, i.e., $\eta=0.02$. The GS configurations for the system with $N=50$ particles and anisotropy parameters $\alpha=0.15,0.3,0.5,0.8$, and 0.98 are shown in Figs. 10(a)-10(e), respectively. Black (red) balls represent particles in the external (inside the external) shell. Figure 10(a) displays a multiple ring structure with particle arrangement $\quad[1:(5 \times) 3:(1,3): 3: 3: 1: 3: 3: 1:(5 \times) 3: 1]$ while Fig. 10(b) does not present any symmetry pattern with respect to rotation around the $z$ direction. The other three GS configurations shown in Figs. 10(c)-10(e) exhibit a multiple ring structure for the internal shell (see red balls) with particle arrangement (1:3:3:1), (1:4:4:1), and (3:3:3), respectively. However, we can notice that the external shells for those GS configurations are not multiple rings.

The fraction of degenerate frequencies for the system with $N=60$ particles and $\alpha=0.25$ is relatively large, that is, $\eta$ $=0.52$ (Fig. 9) and its GS configuration corresponds to a multiple ring structure of arrangement $[1:(2 \times) 4: 1:(2$ ×) $4: 1: 4:(1,4): 4:(1,4): 4: 1:(2 \times) 4: 1:(2 \times) 4: 1]$ which is shown in Fig. 10(f). In contrary, the value of the frequencies for the system with $N=70$ particles is small $(\eta=0.02)$ throughout all the range of the anisotropy parameter and this system does not present multiple ring structure.

By decreasing the anisotropy parameter $\alpha$ one extends the cluster along the $z$ direction. In fact, we can see for the system with $N=50$ particles that the length scale shown in the top right of each GS configuration in Fig. 10 decreases from Figs. 10(a)-10(e). The increase of the particle density forces the formation of a triangular arrangement of particles on the external shell in order to reduce the interparticle interaction energy. Such a triangular arrangement of particles constitutes the preferable configuration, i.e., the configura- 


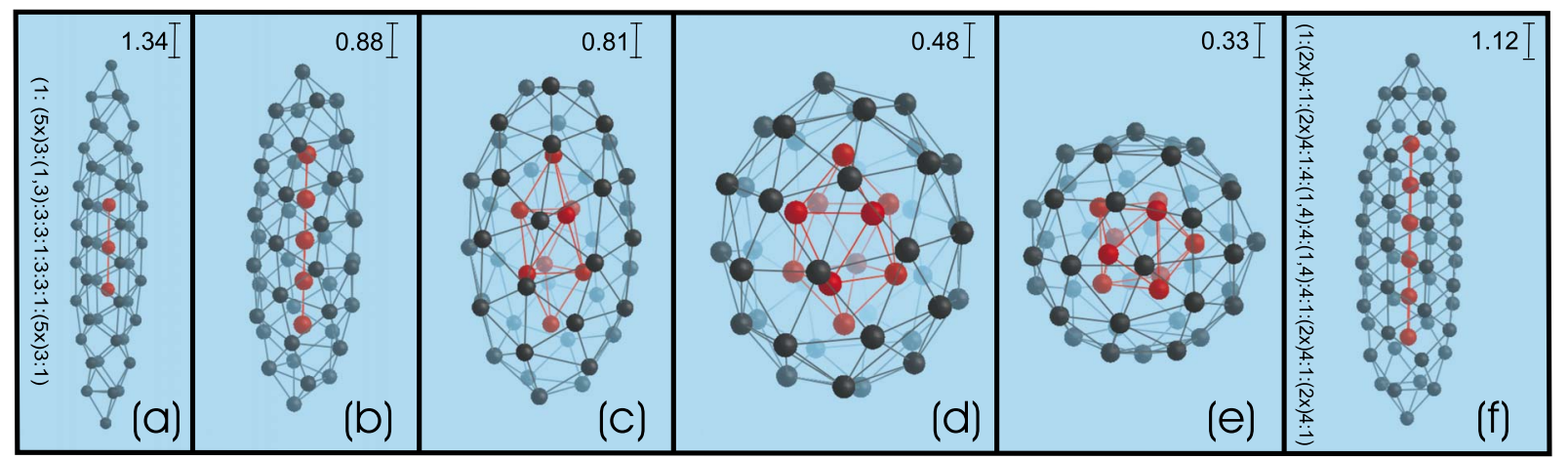

FIG. 10. (Color online) From (a) to (e) the GS configurations for systems with $N=50$ particles and anisotropy parameter $\alpha=0.15,0.3,0.5$, 0.8 , and 0.98 , respectively. (f) GS configuration for the system with $N=60$ particles and $\alpha=0.25$. Black (red) balls correspond to particles in the external (inside the external) shell. The length unit is indicated on the top right of each figure.

tion of lowest energy, in a $2 \mathrm{D}$ infinity system of repulsively interacting particles.

As a result, multiple ring structure of the whole cluster is not formed in large systems under slight anisotropic confinement. Nevertheless, particles in the internal shell are still able to self-organize in multiple rings for the system with $N=50$ particles. In contrast, this is not seen for systems with $N=60$ and 70 particles. This indicates that the formation of multiple ring structure of the internal shell is gradually lost as the number of particles increases. In other words, for large systems in the presence of a confinement potential, the GS becomes a nonsymmetric configuration. This is also supported by the fact that the increase of the number particles leads to the formation of a bcc arrangement which is the preferable particle arrangement for a 3D infinite system. In the limit of a large number of particles the shell structure becomes only well defined at the border of the cluster. However, for partial multiple ring configurations, that is, multiple ring uniquely present in the internal shell, the fraction of degenerate frequencies is no longer a good symmetry indicator. In other words, it does not always differentiate clusters with multiple rings in the internal shell from ordinary nonsymmetric configurations, although such an indicator works properly for multiple ring structures as we saw for the clusters with $N=50$ and 60 particles, and anisotropy parameter $\alpha=0.15$ and 0.25 , respectively.

\section{NORMAL MODES}

The two-dimensional character of the dynamics in a multiple ring structure, that is, oscillations in the plane of the rings, becomes visible via a normal mode analysis. Moreover, the low-energy modes can suggest melting behavior. ${ }^{25,27}$ We display in Fig. 11 the eigenvectors associated with the first nine lowest eigenfrequencies of the system with $N=18$ particles and anisotropy parameter $\alpha=0.4$. The rotation mode around the $z$ axis which has frequency equal to zero is shown in Fig. 11(a). The eigenvectors shown in Figs. $11(\mathrm{~b})$ and $11(\mathrm{c})$ are degenerate and have a frequency equal to $\omega=0.16985$. We notice that for this mode particles' oscillations correspond to two-dimensional dynamics. It is well known that the rotation mode in an isotropically confined system is threefold degenerate and has frequency equal to zero. If the confinement potential is anisotropic some of those degenerate modes obtain a frequency different from zero. Such a mode is seen in Figs. 11(d) and 11(e), which display twofold degenerate rotation modes of nonzero frequency $\omega=0.32651$. Such oscillation corresponds to a rotation mode around a given axis in the $x y$ plane.

The oscillation mode shown in Fig. 11(f) is an inter-ring rotation mode and has frequency $\omega=0.38892$. Notice that the amplitude of oscillation in the two external rings is much larger than the one in the two most internal rings. In what concerns the direction of oscillation we can see that the two upper rings oscillate out of phase with respect to the two lower rings. The seventh and eight modes [see Figs. 11(g)

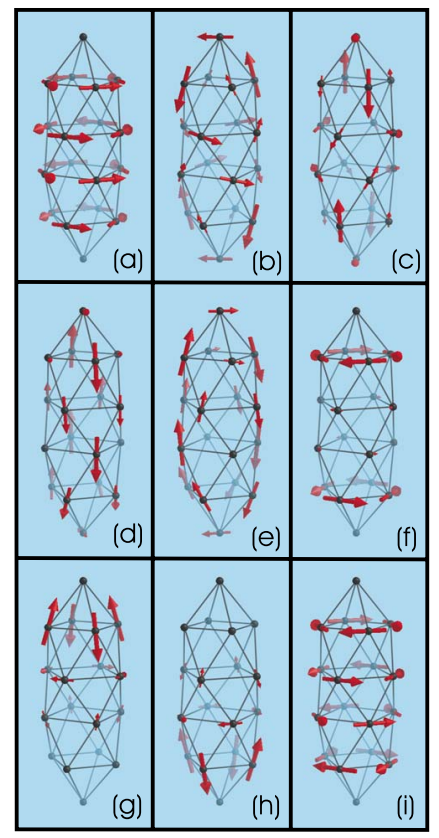

FIG. 11. (Color online) From (a) to (i) we display the first nine lowest-energy eigenvector oscillation modes for the system with $N=18$ particles and $\alpha=0.4$. The direction and length of the vectors indicate, respectively, the direction and amplitude of oscillation of each particle in the cluster. 


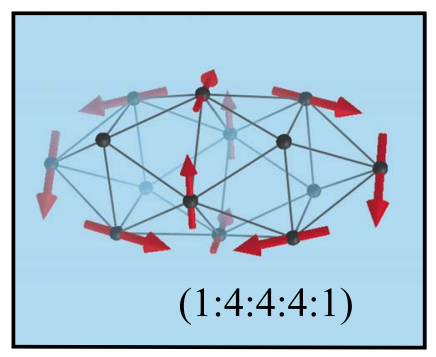

FIG. 12. (Color online) Eigenvector associated with the fourth frequency $\omega=0.3786$ for the system with $N=14$ particles and anisotropy parameter $\alpha=0.5$. This mode corresponds to a vortex/ antvortex motion.

and 11(h)] are degenerate and have frequency equal to $\omega$ $=0.39401$. We can see that the amplitude of oscillations is much larger for those particles close to the extreme of the cluster. The latter fact is indicative for the appearance of inhomogeneous melting. ${ }^{27}$ Finally, Fig. 11(f) displays the eigenvector associated with the frequency $\omega=0.61093$. This oscillation mode corresponds to an inter-ring motion around the $z$ axis. We notice that the two most external (internal) rings oscillate clockwise (anticlockwise). The inter-ring rotation modes shown in Figs. 11(f) and 11(i) indicate that the low temperature dynamics in multiple ring structure must have a strong two-dimensional behavior.

Such inter-ring oscillation modes are not only found for $N=18$ and $\alpha=0.4$, but we also found it for all multiple ring configurations that we presented in Fig. 7. Nevertheless some of the configurations exhibit normal modes that correspond to a vortex/antivortex excitation around axes perpendicular to the $z$ direction, see, for example, Fig. 12 for $N$ $=14$ particles and anisotropy parameter $\alpha=0.28$. In such situations, full 3D melting is expected. Interestingly, vortex/ antvortex motion appears for multiple ring structure of systems with a relatively small number of particles, i.e., $N=14$ particles, while that in 2D clusters vortex/antvortex motion typically appears for large systems, i.e., roughly $N>40$ particles. $^{30,31}$

\section{CONCLUSION}

In summary we have investigated a 3D system of equally charged particles confined anisotropically by an external confinement potential. We assumed that the particles interact via a Coulombic interparticle interaction potential. Our theoretical model is applicable for systems such as dusty plasma and colloids. It is expected that the found behavior is qualitatively the same for other isotropic interaction potentials as, e.g., a screened Coulomb potential. We found that the anisotropy parameter $\alpha$ can drive the system to undergo first and second order structural phase transitions. Those transitions are found to affect the distribution of particles along the $z$ direction and the eigenfrequency spectrum.

The system was found to self-organize in three different general structures, i.e., multiple rings, degenerate multiple rings, and nonsymmetric structures, if the number of particles is small, typically $N \leqslant 25$ particles. For larger systems, i.e., $N \geqslant 50$, multiple ring structures were found in the external shell only if the anisotropy parameter was smaller than $\alpha \leqslant 0.2$, or in the internal shell for more isotropic confinements, roughly $\alpha \geqslant 0.4$. A fine structure was formed in small clusters upon increasing the particle number. Our results on the structure of the GS configurations as function of the anisotropy parameter were summarized in a phase diagram.

For a multiple ring structure the GS configuration is found to exhibit inter-ring and vortex/antivortex modes of oscillation. The latter modes are delocalized and therefore must be of relevance for the melting process. Furthermore, those modes indicate that low temperature dynamics in multiple ring structures must have a strong $2 \mathrm{D}$ behavior. Next to it we found that for the multiple ring configurations the eigenfrequency spectrum becomes highly degenerate. Oppositely, nonsymmetric systems are found to have a low degenerate eigenfrequency spectrum. For the latter case the pattern of oscillation of the eigenvectors become a much more complex and therefore it is impossible to give general trends as function of the anisotropy parameter.

Finally, we notice that the identified multiple ring structure is relevant for the physics of small Coulomb crystals due to the fact that they constitute a hybrid system built up by blocks of 2D structures forming piled rings along the $z$ direction. We expect that the melting process of such a system will follow from the interplay between the dynamics of both a 2D and 3D isotropically confined Coulomb clusters.

\section{ACKNOWLEDGMENT}

This work was supported by the Flemish Science Foundation (FWO-Vl).

\footnotetext{
*sergio.apolinario@ua.ac.be

†bart.partoens@ua.ac.be

*francois.peeters@ua.ac.be

${ }^{1}$ C. C. Grimes and G. Adams, Phys. Rev. Lett. 42, 795 (1979).

${ }^{2}$ E. Y. Andrei, G. Deville, D. C. Glattli, F. I. B. Williams, E. Paris, and B. Etienne, Phys. Rev. Lett. 60, 2765 (1988).

${ }^{3}$ J. H. Chu and Lin I, Phys. Rev. Lett. 72, 4009 (1994).

${ }^{4}$ Y. Kondo, J. S. Korhonen, M. Krusius, V. V. Dmitriev, E. V. Thuneberg, and G. E. Volovik, Phys. Rev. Lett. 68, 3331 (1992).
}

${ }^{5}$ W. M. Itano, J. J. Bollinger, J. N. Tan, B. Jelenković, X.-P. Huang, and D. J. Wineland, Science 279, 686 (1998).

${ }^{6}$ L. Hornekær, N. Kjærgaard, A. M. Thommesen, and M. Drewsen, Phys. Rev. Lett. 86, 1994 (2001).

${ }^{7}$ O. Arp, D. Block, A. Piel, and A. Melzer, Phys. Rev. Lett. 93, 165004 (2004).

${ }^{8}$ J. E. Hug, F. van Swol, and C. F. Zukoski, Langmuir 11, 111 (1995).

${ }^{9}$ S. Neser, T. Palberg, C. Blechinger, and P. Leiderer, Prog. Colloid 
Polym. Sci. 104, 194 (1997).

${ }^{10}$ A. Melzer, A. Homann, and A. Piel, Phys. Rev. E 53, 2757 (1996).

${ }^{11}$ H. Thomas and G. E. Morfill, Nature (London) 379, 806 (1996).

${ }^{12}$ L. I, W.-T. Juan, C.-H. Chiang, and J. H. Chu, Science 272, 1626 (1996).

${ }^{13}$ A. Piel and A. Melzer, Plasma Phys. Controlled Fusion 44, R1 (2002).

${ }^{14}$ W.-T. Juan, Z.-H. Huang, J.-W. Hsu, Y.-J. Lai, and L. I, Phys. Rev. E 58, R6947 (1998).

${ }^{15}$ M. Klindworth, A. Melzer, A. Piel, and V. A. Schweigert, Phys. Rev. B 61, 8404 (2000).

${ }^{16}$ G. E. Morfill, H. M. Thomas, U. Konopka, H. Rothermel, M. Zuzic, A. Ivlev, and J. Goree, Phys. Rev. Lett. 83, 1598 (1999).

${ }^{17}$ A. P. Nefedov, G. E. Morfill, V. E. Fortov, H. M. Thomas, H. Rothermel, T. Hagl, A. V. Ivlev, M. Zuzic, B. A. Klumov, A. M. Lipaev, V. I. Molotkov, O. F. Petrov, Y. P. Gidzenko, S. K. Krikalev, W. Shepherd, A. I. Ivanov, M. Roth, H. Binnenbruck, J. A. Goree, and Y. P. Semenov, New J. Phys. 5, 33.1 (2003).

${ }^{18}$ M. Klindworth, A. Piel, A. Melzer, U. Konopka, H. Rothermel, K. Tarontik, and G. E. Morfill, Phys. Rev. Lett. 93, 195002 (2004).

${ }^{19}$ M. Klindworth, O. Arp, and A. Piel, J. Phys. D 39, 1095 (2006).

${ }^{20}$ M. Bonitz, D. Block, O. Arp, V. Golubnychiy, H. Baumgartner, P. Ludwig, A. Piel, and A. Filinov, Phys. Rev. Lett. 96, 075001 (2006).
${ }^{21}$ P. Ludwig, S. Kosse, and M. Bonitz, Phys. Rev. E 71, 046403 (2005).

${ }^{22}$ V. Golubnychiy, H. Baumgartner, M. Bonitz, A. Filinov, and H. Fehske, J. Phys. A 39, 4527 (2006).

${ }^{23}$ H. Löwen, Phys. Rep. 237, 249 (1994).

${ }^{24}$ S. W. S. Apolinario, B. Partoens, and F. M. Peeters, New J. Phys. 9, 283 (2007).

${ }^{25}$ S. W. S. Apolinario and F. M. Peeters, Phys. Rev. E 76, 031107 (2007).

${ }^{26}$ S. W. S. Apolinario, B. Partoens, and F. M. Peeters, Phys. Rev. E 72, 046122 (2005).

${ }^{27}$ S. W. S. Apolinario, B. Partoens, and F. M. Peeters, Phys. Rev. E 74, 031107 (2006).

${ }^{28}$ G. Piacente, I. V. Schweigert, J. J. Betouras, and F. M. Peeters, Phys. Rev. B 69, 045324 (2004).

${ }^{29}$ Y. G. Cornelissens, B. Partoens, and F. M. Peeters, Physica E (Amsterdam) 8, 314 (2000).

${ }^{30}$ V. A. Schweigert and F. M. Peeters, Phys. Rev. B 51, 7700 (1995).

${ }^{31}$ Minghui Kong, B. Partoens, A. Matulis, and F. M. Peeters, Phys. Rev. E 69, 036412 (2004).

${ }^{32}$ B. Partoens, V. A. Schweigert, and F. M. Peeters, Phys. Rev. Lett. 79, 3990 (1997).

${ }^{33}$ K. Tsuruta and S. Ichimaru, Phys. Rev. A 48, 1339 (1993).

${ }^{34}$ D. M. Tomecka, B. Partoens, and F. M. Peeters, Phys. Rev. E 71, 062401 (2005). 